they promote proliferation in cancer cells. Mutations in BRaf are commonly found in cancer, and drugs targeting the enzyme are in clinical use. Of these, the type I inhibitor, SB590885 (SB), binds to the active conformation of BRaf, whilst dabrafenib has no activation state selectivity. Paradoxically, these inhibitors activate ERK1/2 in cancer cells by stimulating the cascade at low concentrations rather than inhibiting it. Our aim is to determine the effects of BRaf inhibitors on the ERK1/2 cascade in cardiomyocytes to establish whether they may activate the pathway in the hearts of patients undergoing cancer therapy.

Methods Rat neonatal cardiomyocytes were exposed to dabrafenib or $\mathrm{SB}$ and the effects on activation of $\mathrm{MKK} 1 / 2$ or ERK1/2 examined by immunoblotting with antibodies to phosphorylated (i.e. activated) or total kinases. Raf activities were assayed using GST-MKK1 as a substrate. The effects on myocyte morphology were studied by immunostaining for myofibrillar proteins.

Results Low concentrations of SB $(0.1 \mu \mathrm{M})$ or dabrafenib (1 $\mu \mathrm{M}$ ) enhanced ERK1/2 signalling, with SB having a greater effect than dabrafenib. Thus, cardiomyocytes are primed for paradoxical activation of ERK1/2 by BRaf inhibitors. One explanation for the Raf paradox is that BRaf forms homo or heterodimers with cRaf. Thus, a submaximal concentration of BRaf inhibitor may be sufficient to inhibit only one Raf protein and, since the kinase is locked in an active state, there is activation of the other Raf partner. Consistent with this, 0.1 $\mu \mathrm{M}$ SB increased activities of both BRaf and cRaf, although there was no significant increase in dimerization. High concentrations of SB or dabrafenib $(1-10 \mu \mathrm{M})$ inhibited basal ERK1/ 2 activity in cardiomyocytes, but the inhibitory effect was only transient and levels returned to basal within 1 hour. Furthermore, high concentrations of SB activated ERK1/2 over prolonged periods (up to 24 hour). This was associated with an increase in cardiomyocyte size and myofibrillar organisation consistent with low level activation of the pathway promoting cardiomyocyte hypertrophy. In mouse hearts in vivo, dabrafenib and SB590885 enhanced MKK1/2 phosphorylation over 24 hour, and increased hypertrophic ANF and BNP mRNA expression markers, consistent with the cell data.

Conclusions Cardiomyocytes are primed for Raf paradox signalling and BRaf inhibitors such as SB and dabrafenib activate ERK1/2 in cardiomyocytes. Consistent with a role for ERK1/2 in cardiomyocyte hypertrophy, SB increases expression of hypertrophic gene markers and promotes morphological changes associated with hypertrophy.

\section{ROLE OF SUMOYLATION AND DESUMOYLATION OF MITOCHONDRIAL FISSION PROTEINS IN MYOCARDIAL ISCHAEMIA-REPERFUSION INJURY}

${ }^{1}$ Nadiia Rawlings*, ${ }^{1}$ Laura Lee, ${ }^{2}$ Jordan Martin, ${ }^{1}$ Richard Seager, ${ }^{3}$ Chun Guo, ${ }^{1}$ Kevin Wilkinson, ${ }^{1}$ Andrew Halestrap, ${ }^{1}$ Jeremy Henley. ' University of Bristol; ${ }^{2}$ University of Plymouth; ${ }^{3}$ University of Sheffield

\subsection{6/heartjnl-2017-311726.227}

Background Restoration of blood supply to the heart after a prolonged ischaemic episode causes myocardial ischaemiareperfusion (I/R) injury. Paradoxically, short periods of ischaemia and reperfusion, known as preconditioning, has a potent cardioprotective effect. One key aspect of $\mathrm{I} / \mathrm{R}$ injury is cytotoxic mitochondrial fission that allows cytochrome $c$ release and promotes apoptotic cell death. Dynamin-related protein 1
(Drp1) is a large GTPase that is recruited from the cytosol to the mitochondrial outer membrane to drive fission. Drp1 function is regulated by posttranslational modifications, including conjugation to small ubiquitin-like modifier (SUMO)-2/3. SUMO-2/3-ylation decreases Drp1 partitioning to the mitochondrial membrane, which reduces stress-induced fission and apoptosis. We have shown previously that, in neurons, Drp1 SUMO-2/3-ylation is controlled by the deSUMOylating enzyme SENP3, levels of which are reduced during ischaemia but restored upon reperfusion.

Objective Here we investigated alterations in SUMOylation and Drp1 partitioning in heart during ischaemia, I/R and preconditioning. Methods: Isolated hearts from male Wistar rats were perfused using Langendorff apparatus with Krebs Henseleit solution. Hearts were randomly divided into 4 groups (with $\mathrm{n}=5-6$ per group): control (50 min perfusion); ischaemia (20 min perfusion +30 min ischaemia); preconditioning (3 short cycles of I/R, 2 and 3 mins, respectively, followed by $30 \mathrm{~min}$ of ischaemia); $I / R$ (30 min of ischaemia followed by 2 hour of reperfusion). All samples were immediately subjected to subcellular fractionation, then frozen on dry ice and used for Western blot analysis.

$1 \mathrm{~A}$

Total SENP3.

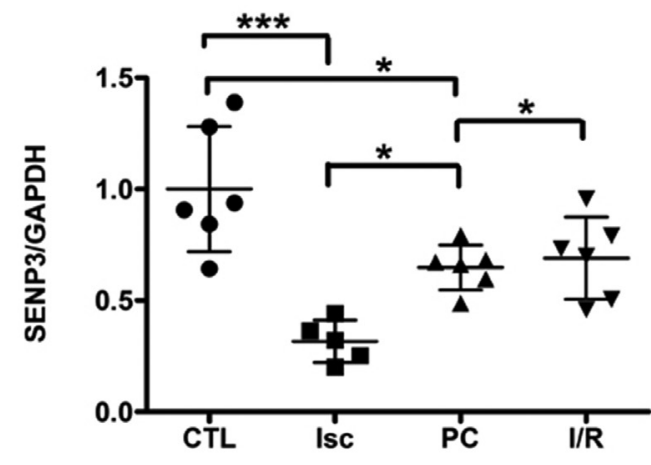

1B

Total SUMO-2/3-ylation. Mitochondrial fraction.

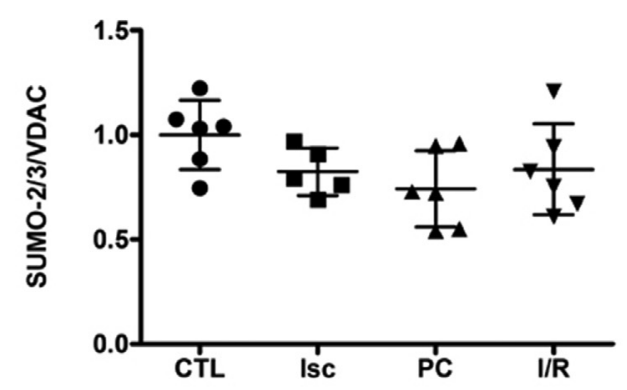

Abstract 229 Figure 1

Results Levels of SENP3 were reduced in all three groups compared to control, with the greatest reduction seen during ischaemia (Fig 1A). Despite the observed reduction in SENP3 levels no obvious change in overall levels of protein SUMO-2/ 3 -ylation was detected in mitochondria fractions (Fig 1B). An increase of SUMO-1 conjugation to an unidentified protein at about $55 \mathrm{kDa}$ was observed during ischaemia and $\mathrm{I} / \mathrm{R}$ 
compared to both the control and preconditioning groups (Fig 2A). Intriguingly, in contrast to our finding in neurons, where mitochondrial partitioning of Drp1 decreases during ischaemia, in cardiac tissue we observed recruitment of Drp1 to mitochondria, with no change in total protein levels. Furthermore, Drp1 recruitment to mitochondria was increased by preconditioning. In the $\mathrm{I} / \mathrm{R}$ group, in which cells are undergoing apoptosis, levels of Drp1 at the mitochondria are similar to controls (Fig 2B).

\section{A SUMO-1-ylation of $\approx 55 \mathrm{kDa}$ band. Mitochondrial fraction.}

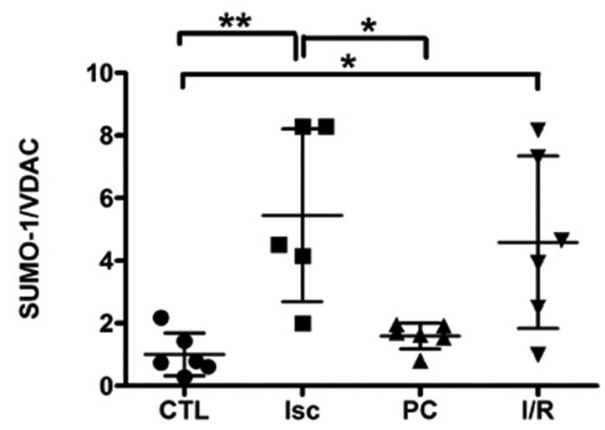

2B

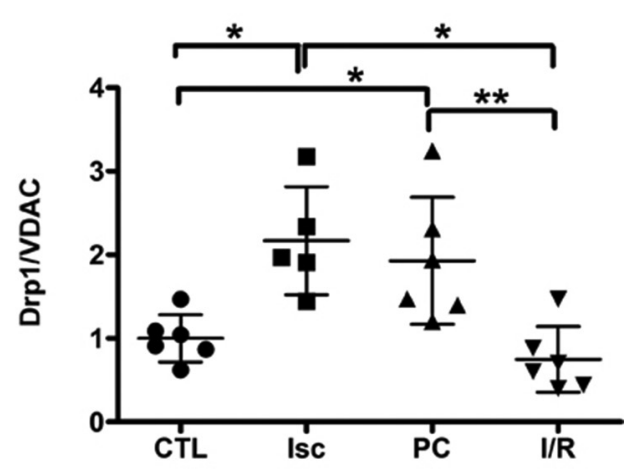

Abstract 229 Figure 2

Conclusion Taken together our data suggest a delicate balance between SUMOylation and deSUMOylation that regulates the recruitment of Drp1 to mitochondria. This pathway plays an important role in the vulnerability of cardiomyocytes to ischaemic damage and myocardial reperfusion injury. Interestingly, the interplay between the relevant proteins appears to differ between heart and brain cells.

\section{TRACKING LATE OUTGROWTH ENDOTHELIAL CELLS IN AN ACUTE ARTERIAL INJURY MODEL}

Takeshi Fujisawa*, Andrew Mitchell, Claire Medine, Carlos Alcaide Corral, Adriana Tavares, Nicholas L Mills, Patrick WF Hadoke. BHF/University Centre for Cardiovascular Science, University of Edinburgh

\subsection{6/heartjnl-2017-311726.228}

Aim Late outgrowth endothelial cells (EOC) are strong contenders to be the true circulating endothelial progenitor cells since they are capable of clonogenic expansion, exhibit a mature endothelial phenotype, and contribute to angiogenesis in vivo. These cells may play a crucial role in the process of vascular repair, but whether they are able to accumulate at sites of vascular damage in vivo is not clear. We hypothesise that EOC, delivered locally or systemically, accumulate at, and incorporate into, a site of arterial injury.

Methods Experimental groups comprised systemic administration of the glucose analogue radiotracer ${ }^{18} \mathrm{~F}$-Flurodeoxyglucose (FDG) (Sys-Free) or FDG-labelled EOC (Sys-EOC), or local administration of free FDG (Local-Free) or FDG-labelled EOC (Local-EOC). EOC were isolated from peripheral blood from patients with coronary heart disease $(n=3)$. Left femoral artery injury was achieved in male Sprague Dawley rats (300-350g) under general anaesthesia by inserting micro-renathane tubing via the popliteal artery. EOC were labelled with FDG (25 $\mathrm{MBq} / \mathrm{ml}, 30 \mathrm{~min}, 37^{\circ} \mathrm{C}$ ) and 1 million cells were administered either locally into the femoral artery or systemically via the tail vein (0.3-1.3 MBq, $\mathrm{n}=3$ per group). Following injection of radiolabelled cells or free FDG, rats underwent dynamic PET scanning over 4 hours (Mediso nanoPET/CT scanner, Hungary). AuroVist and Fenestra (MediLumine Inc, Canada) were used as computed tomography vascular contrast agents. Images were analysed with PMOD software (PMOD, Switzerland) and standardised uptake values were calculated.

Results FDG radioactivity was successfully visualised by microPET/CT. The activity was distributed in the bladder, kidneys, heart, brain, lungs, spleen and liver (descending order). Radioactivity in the lungs was significantly higher (80 and $120 \mathrm{~min}$ ) following systemic EOC administration compared with the other three groups (two-way ANOVA with Bonferroni posttest, $\mathrm{p}<0.001$ ), and peak activity in the injured artery $(55 \mathrm{~min}$ after administration) was significantly higher than in the noninjured right artery (two-way ANOVA with Bonferroni posttest, $\mathrm{p}<0.001)$. Following local EOC administration, radioactivity in the injured artery was significantly higher than in the non-injured right artery, or in the injured artery following local free FDG administration (two-way ANOVA with Bonferroni post-test, $\mathrm{p}<0.001)$. Radioactivity in the injured artery following local administration was considerably higher $(\sim 10$ fold $)$ than following systemic administration of either free FDG or labelled EOC.

Conclusion Preliminary analysis shows that EOC are able to target sites of vascular injury following systemic and local administration. These observations suggest that late outgrowth endothelial cells have the potential to contribute to vascular repair and regeneration.

\section{SUPER RESOLUTION IMAGING UNVEILING THE DYADIC ULTRASTRUCTURE IN ATRIAL AND VENTRICULAR CARDIOMYOCYTES}

Lauren Toms*, Jessica Caldwell, Andrew Trafford, Katharine Dibb. University of Manchester

10.1136/heartjnl-2017-311726.229

Calcium-induced calcium release drives contraction in cardiomyocytes. Located on the sarcoplasmic reticulum, ryanodine receptors (RyR) are responsible for the release of intracellular calcium stores. It has recently become apparent that the size and shape of RyR cluster may affect the functionality of that cluster, along with the relative distance to neighbouring clusters. Here, we compared the distribution of RyRs in the atria and ventricle. 